\title{
The Research of Power Frequency Interference in the Acquisition System of SEMG
}

\author{
Hanqing Zhao ${ }^{1,2}$, Bo You ${ }^{3}$, Yi Liu ${ }^{3}$ and Shoutong $\mathrm{Tao}^{3}$ \\ ${ }^{1}$ Collge of Measure-Control Technology and Communication Engineering, \\ Harbin University of Science and Technology, Harbin, Chna \\ ${ }^{2}$ Collge of Mechanical Engineering, \\ Heilongjiang Institute of Science and Technology, Harbin, China \\ ${ }^{3}$ Collge of Automation, Harbin University of Science and Technology, Harbin, Chna \\ Zhaohanqing0001@163.com \\ youbo@hrbust.edu.cn
}

\begin{abstract}
The power frequency in $50 \mathrm{~Hz}$ and its harmonic interference is one of the main noise sources during the acquisition process of surface electromyogram signals (SEMG), how to restrain interference power frequency and its harmonics effectively is one of the important issues on the EMG preprocessing. In this paper, we will determine the power frequency interference and its frequency of the harmonic through the signal acquisition, make the crosscorrelation by the constructor and the collected data, and determine its magnitude. We can draw a conclusion that this method can effectively restrain power frequency interference of EMG during the acquisition process through the comparison of the power spectrum of denoising before and after. The Experiments show that this method can effectively separate the power frequency interference during the acquisition process, and is suitable for the real-time processing of EMG.
\end{abstract}

Keywords: SEMG; power frequency interference; associated estimates; spectral analysis

\section{Introduction}

The SEMG is a type of bio-electricity signal along with the body movements, it has been widely used in the field of clinical medicine, sports medicine, bionic limbs and medical rehabilitation, as the physiological status of muscle, the degree of muscle fatigue and the human body motion can be studied through the research [1]. In the system of EMG acquisition interface, we can abstract the characteristic value and control associated equipment by obtaining the SEMG from the human body. The system of EMG acquisition interface needs to be used in the general places finally, so the system with the nature of simpleness, fast progress, convenient, reliability and easy to be accepted by users is the development tendency in the future [2].

The SEMG is a feeble physiology signals (only with the level of millivolt), the system of EMG acquisition interface can easily been polluted by the noise, especially the industrial frequency noise $[3,4]$. So how to restrain and reduce electric power frequency interference is an important research subject during the SEMG collection of the human-machine interface. At present, we mainly use notch processing to dispose the power frequency interference, so the wave trap of power frequency interference can be widely used in the field of data collection and analysis, and as the classic IIR wave filter has a nonlinear phase, is adaptive 
filtering and the number of wavelet analysis is too large to be calculated [5, 6]. So we can only use FIR wave filter in practice, but we have to use a higher order to obtain a better fading characteristic, and it will result in the difficulty of the hardware processing $[7,8]$. In addition, as the frequency spectrum of SEMG and its power frequency interference is mixed, the notching filter will remove the power frequency interference as well as a part of virtual components of SEMG in its working frequency band. The small losses of SEMG will lead to information gap of signal and influence the precision of SEMG pattern recognition. In this paper, we will propose an effective method to remove the power frequency interference and its harmonic interference of SEMG based on the correlation analysis, can filter continuous SEMG of hand motion collected and remove its power frequency interference and its harmonic interference effectively.

\section{The Analysis of Power Frequency Interference Sources}

\subsection{The Composition of SEMG Acquisition System}

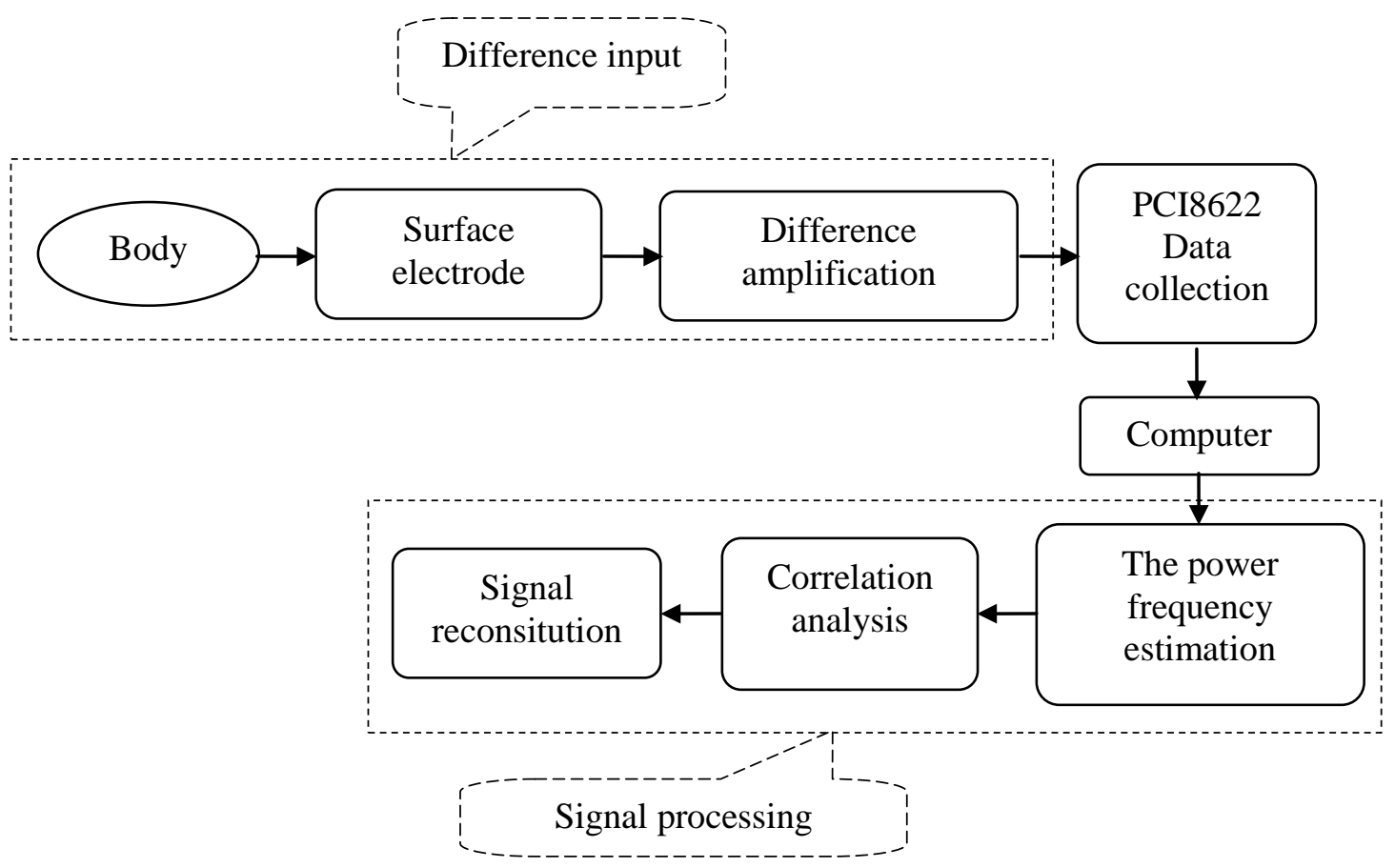

Figure 1. Signal Acquisition and Processing

The SEMG acquisition system can be seen as the figure 1, we can collect the SEMG from volunteers by the surface electrode pasted in the arm, then the signal will be sent to the differential amplification circuit, and then to the computer through the data card, so we can proceed the data preprocessing. In this paper, we can use the PCI8622 Data Acquisition Card product by the Alltel Corp, its AD conversion accuracy is 16 bits, sample frequency is $1 \mathrm{~Hz}-$ $250 \mathrm{kHz}$, analog input channel has double-end 16 channels, gain-programmed amplifier is 1 , $10,100,1000$, digital Output has 16 channels, it can meet the acquisition requirements of SEMG. 


\subsection{The Mechanism of Power Frequency Induction}

The acquisition of SEMG is a type of non-invasive detection method through the surface electrode which can measure the SEMG of human body in different condition, so this method can be easily accepted by users. But this acquisition method has a sophisticated way on the contact of the electrode and skin, in the Figure 2, it can be equaled to a cascade between a LF voltage and a impedance which has correlation frequency normally. The acquisition of EMG has to be interfered by the frequency signal inevitably [8]. As actually the frequency signal interference is about $50 \mathrm{~Hz}$ and also includes its harmonic component. Power frequency interference enter into the detecting system through the induced voltage from human body and distributed capacitance from circuit board produced by electrostatic field and the electromagnetic fields $[9,10]$. As the electro static pressure can be removed by the direct component, so it is generally not considered. In this paper, we can just consider the spread voltage caused by the electromagnetic field. Cpow is the stray capacitance between human body and power line, Cb is the stray capacitance between human body and the earth, Cbo is the stray capacitance between amplifier public side and power line, the stray capacitances are closely related with human body, equipment, power grid and so on, their values can influence the Ac coupling voltage of power frequency from human body and the amplifier public side, the voltage is not the same in the vast majority of cases, their difference is the common-mode voltage. Its peak-to-peak value can reach up to about $10 \mathrm{~V}$, and is much larger than the measured voltage of SEMG. As SEMG has a millivolt level, so needs to be amplified, during this process, the common-mode voltage will cause the equilibration of the component and lead to a result of distortion.

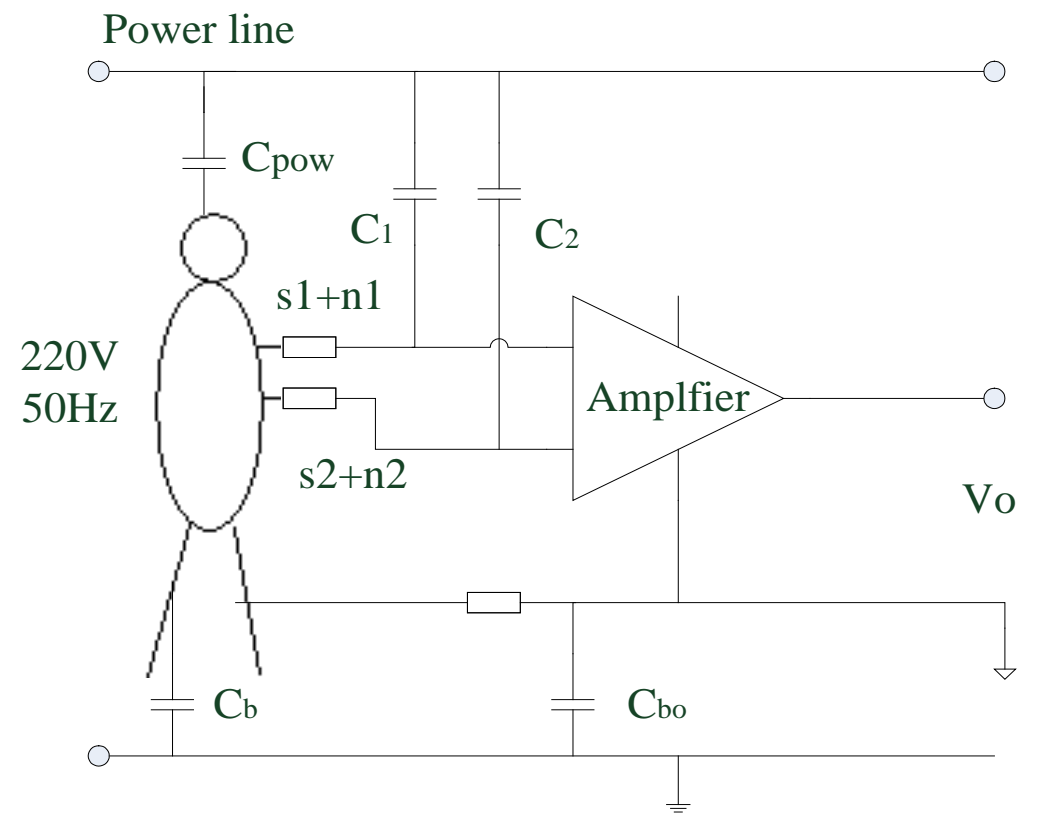

Figure 2. The LNA-IN of EMG in the Power Frequency Interference

\subsection{Hardware Processing Method}

In the field of hardware processing, electromagnetic shielding is an ideal method for the removal of the electromagnetic field interference. We cannot shield the human body totally during the EMG measure, but we can take the appropriate measures to the lead wire and the 
preamplifier, in this course, we will make pre-amplification to a separate circuit board, and then put it into metal carton for the shielding. The pre-amplifier should be close to the electrode as far as possible, electrode unit composed of preamplifier and electrode will improve Snr and would be best. As the distance between the electrodes is close during the signal acquisition, power frequency interference is common mode voltage signal generally, and can be restrained by enhancing common mode rejection capability of the amplification system through the differential circuits.

As the impedance of electrode-skin abutment is mismatched, it will lead to the disaccord between amplifier input signal s1+n1, s2+n2 and its interference signal $n 1$, n2, so output voltage is $V_{O}(t)=s(t)+n(t)$, where the $V_{O}(t)$ is sampling value of SEMG during the time $t, s(t)$ is the actual value of SEMG, $n(t)$ is interference of electric power frequency and its harmonics.

$n(t)$ can be indicated as follow:

$$
n(t)=\sum A_{i} \cos \left(2 \pi f_{i} t+\varphi_{i}\right)
$$

Where $A_{i}$ is the amplitude of the ith harmonic of power frequency electric; $f_{i}$ is the frequency of the ith harmonic of power frequency electric; $\varphi_{i}$ is the phase of the ith harmonic of power frequency electric.

\section{The Principle of Power Frequency Test}

\subsection{The Estimate of Noise Frequency}

We should ensure the EMG value $s(t)$ through the power frequency interference value $n(t)$, we can seen from the formula(2) that we need to ensure the frequency, amplitude and phase of power frequency interference $n(t)$.

First we will calculate the frequency spectrum of $V_{O}(t)$ by the fourier transform[11]

$$
V_{O}(\omega)=F^{-1}\left[V_{O}(t)\right]=\int_{-\infty}^{+\infty} f(t) e^{-j \omega t} d t
$$

As $V_{O}(t)$ includes the available EMG $s(t)$ and the power frequency interference $n(t)$, and $s(t)$ is not a periodic signal, $n(t)$ is a periodic signal of $50 \mathrm{~Hz}$ and its harmonic, the frequency spectrum of non-periodic signal is continuous and the frequency spectrum of periodic signal is discrete based on the nature of Fourier transform, so we can abstract the frequency spectrum of periodic signal through the frequency spectrum $V_{O}(\omega)$ of $V_{O}(t)$, and then we can ensure the frequency of $n(t)$.

\subsection{The Amplitude of Signal and its Estimate of the Phase}

We can estimate the power frequency interference $n(t)$ is $50 \mathrm{~Hz}$ and its harmonic frequency can be ensured from the result above. Now we should ensure the amplitude and phase of $n(t)$, first we should construct a function has the same frequency with power frequency interference $n(t)$ :

$$
g(t)=\sum_{i} \cos \left(2 \pi f_{i} t\right)
$$


Calculating the cross-correlation of sampled signal $V_{O}(t)$ and constructor $g(t)$, and then ensuring the amplitude and phase of power frequency interference $n(t)$.

$$
\begin{aligned}
& R_{V g}(\tau)=\lim _{T \rightarrow \infty} \frac{1}{T} \int_{0}^{T} V_{O}(t) g(t+\tau) d t \\
& =\lim _{T \rightarrow \infty} \frac{1}{T} \int_{0}^{T}\left[s(t)+\sum_{i} A_{i} \cos \left(2 \pi f_{i} t+\varphi_{i}\right)\right] \sum_{i} \cos \left(2 \pi f_{i} t\right) d t \\
& =\lim _{T \rightarrow \infty} \frac{1}{T} \int_{0}^{T} s(t) \sum_{i} \cos \left(2 \pi f_{i} t\right) d t \\
& +\lim _{T \rightarrow \infty} \frac{1}{T} \int_{0}^{t} \sum_{i} A_{i} \cos \left(2 \pi f_{i} t+\varphi_{i}\right) \sum_{i} \cos \left(2 \pi f_{i} t\right) d t
\end{aligned}
$$

According to the relevant nature, same frequency is correlated, different frequency is not correlated, we can seen from formula (3):

$$
\begin{aligned}
& R_{V g}(\tau)=\lim _{T \rightarrow \infty} \frac{1}{T} \int_{0}^{T} \sum_{i} A_{i} \cos \left(2 \pi f_{i} t+\varphi_{i}\right) \sum_{i} \cos \left(2 \pi f_{i} t\right) \\
& =\sum_{i} A_{i} \cos \left(2 \pi f_{i} t+\varphi_{i}\right)
\end{aligned}
$$

Compared the formula (4) with formula (2):

$$
\hat{n}(t)=R(t)=\sum_{i} A_{i} \cos \left(2 \pi f_{i} t+\varphi_{i}\right)
$$

Here, we can obtain power frequency and its harmonic interference values $\hat{n(t)}$ of EMG.

Put the formula (5) into formula (2):

$$
\hat{s(t)}=V_{0}(t)-\hat{n(t)}=\sum_{i} A_{i} \cos \left(2 \pi f_{i} t+\varphi_{i}\right)
$$

Then we can estimate EMG $\hat{s(t)}$ without power frequency and its harmonic interference from the EMG $V_{O}(t)$ collected.

\section{Experiment and Analysis}

We can text the SEMG of these three health volunteers from their arms based on Figure 1, the surface electrode we used is silver or silver chloride electrode, Test muscle group is the forearm side wrist extensor. The analysis software we used is MATLAB R2010a. During the experiment, we will stick a pair of surface electrode to the forearm side wrist extensor, and serve as differential input of the EMG acquisition and stick the reference electrode to the wrist, sample frequency is $1000 \mathrm{~Hz}$. We should clean the forearm skin of the volunteers with the alcohol before the experiment and stick the electrode; during the experiment, the hands of volunteers should relax and fist for 20 times. Figure 3 and 4 are the original signal and its spectrum of the movement relax and fisting, we can see that frequency of power frequency and its harmonic interference includes $50 \mathrm{~Hz}, 150 \mathrm{~Hz}, 250 \mathrm{~Hz}, 350 \mathrm{~Hz}, 450 \mathrm{~Hz}$ during the EMG acquisition. So the constructor $g(t)$ is as follow: 
International Journal of Signal Processing, Image Processing and Pattern Recognition Vol.6, No.5 (2013)

$$
g(t)=\sum_{i=1}^{4} \cos 2 \pi \cdot(2 i-1) \cdot 50 t
$$

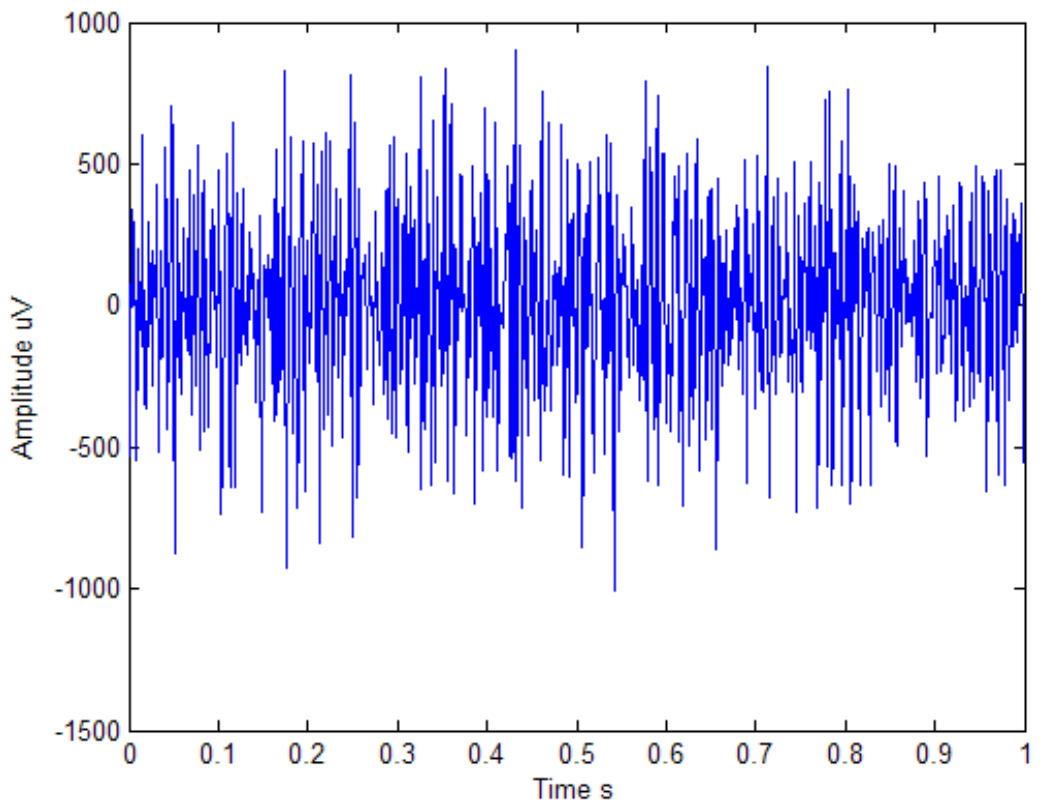

(a) Original Data of Relaxing

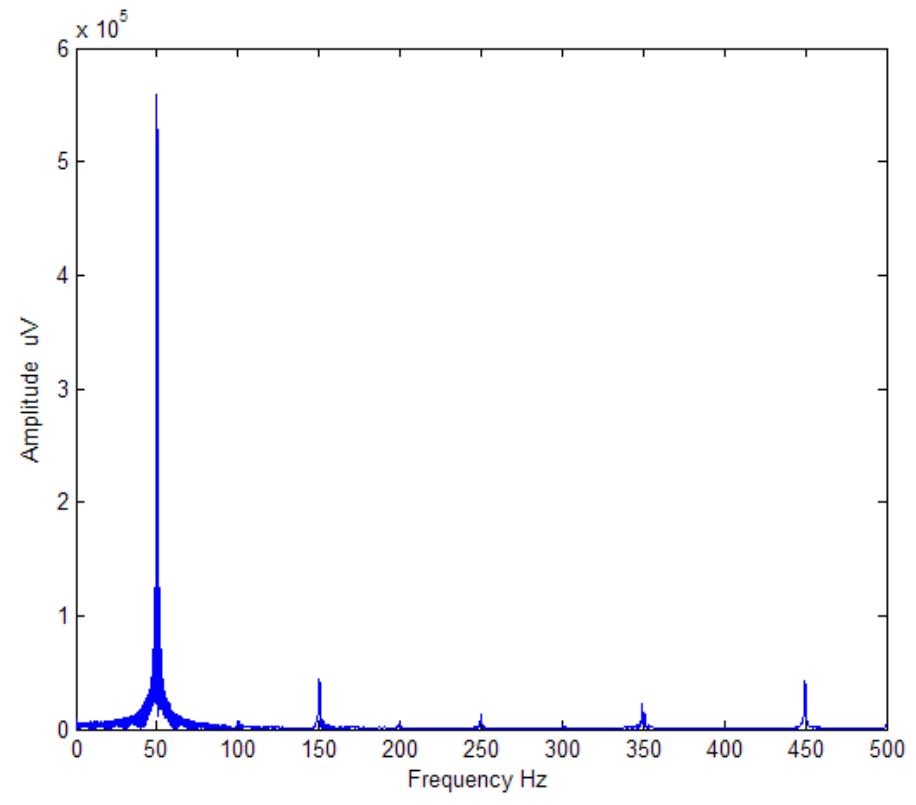

(b) Frequency Spectrum during Relaxing

Figure 3. Observed Signals and its Frequency Spectrum during Relaxing 


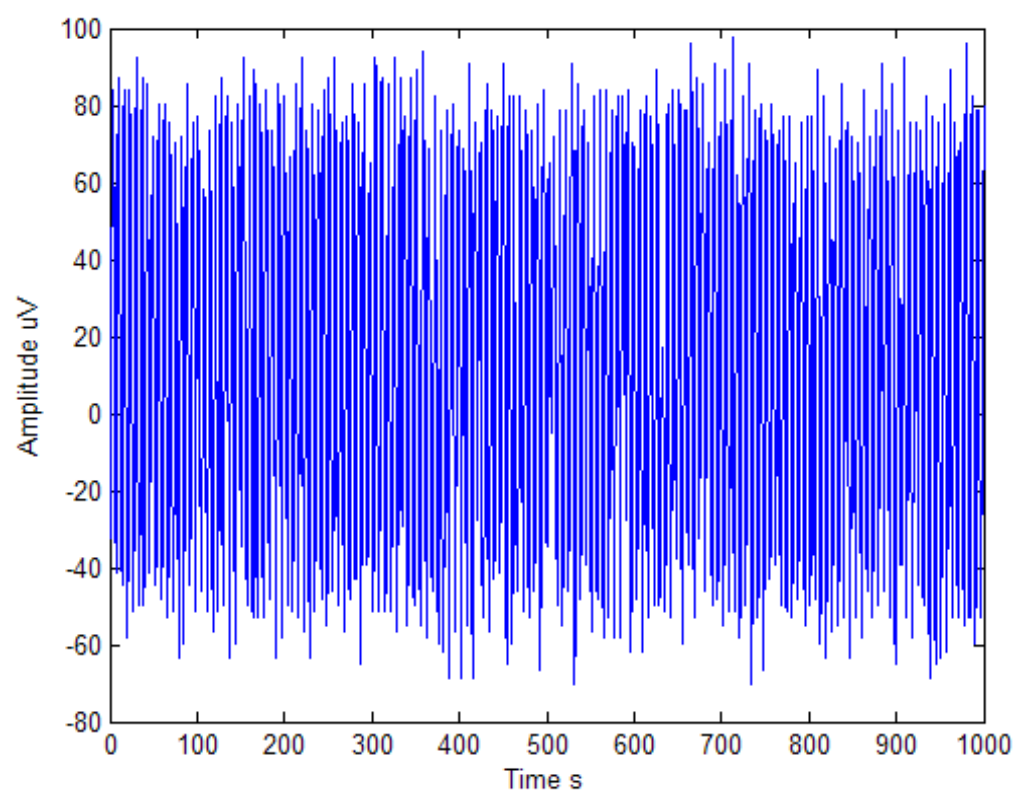

(a) Original Data of Fisting

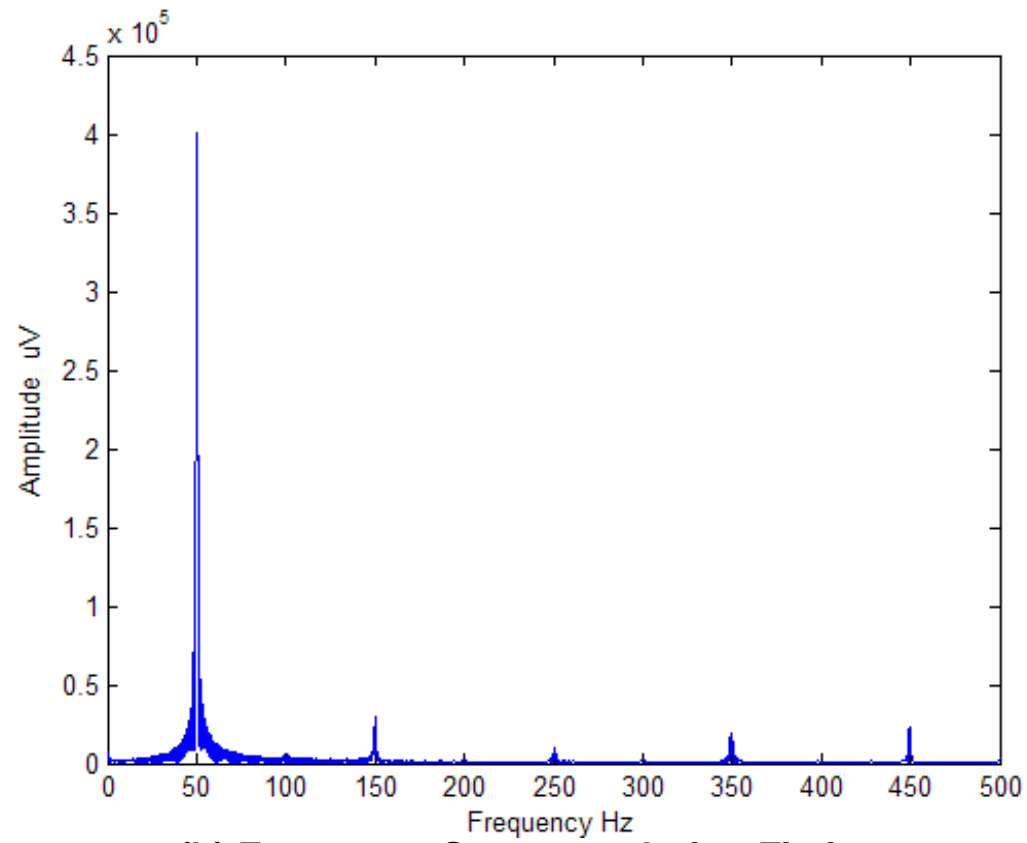

(b) Frequency Spectrum during Fisting

Figure 4. Observed Signals and its Frequency Spectrum during Fisting

We will obtain the EMG values of power frequency and its harmonic interference $n(t)$ through the cross-correlation between constructor $g(t)$ and acquisition signal $V_{O}(t)$, and can get EMG $s(t)$ through formula (5). Figure 5 is the EMG separated. 
International Journal of Signal Processing, Image Processing and Pattern Recognition

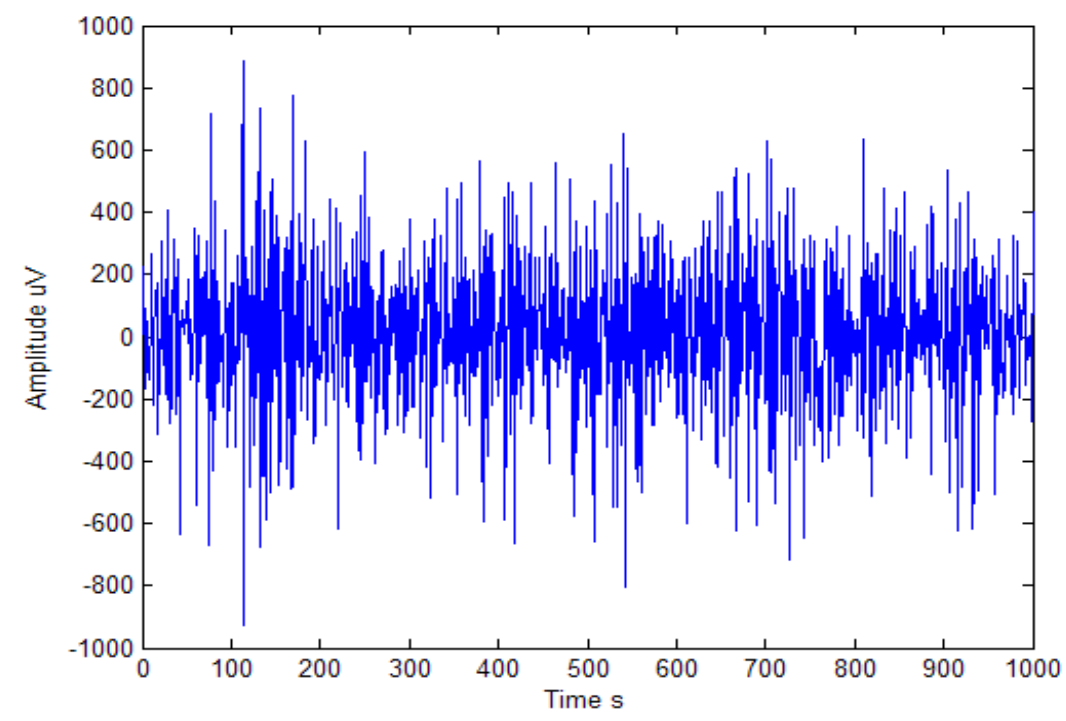

(a) Relaxing EMG Separated

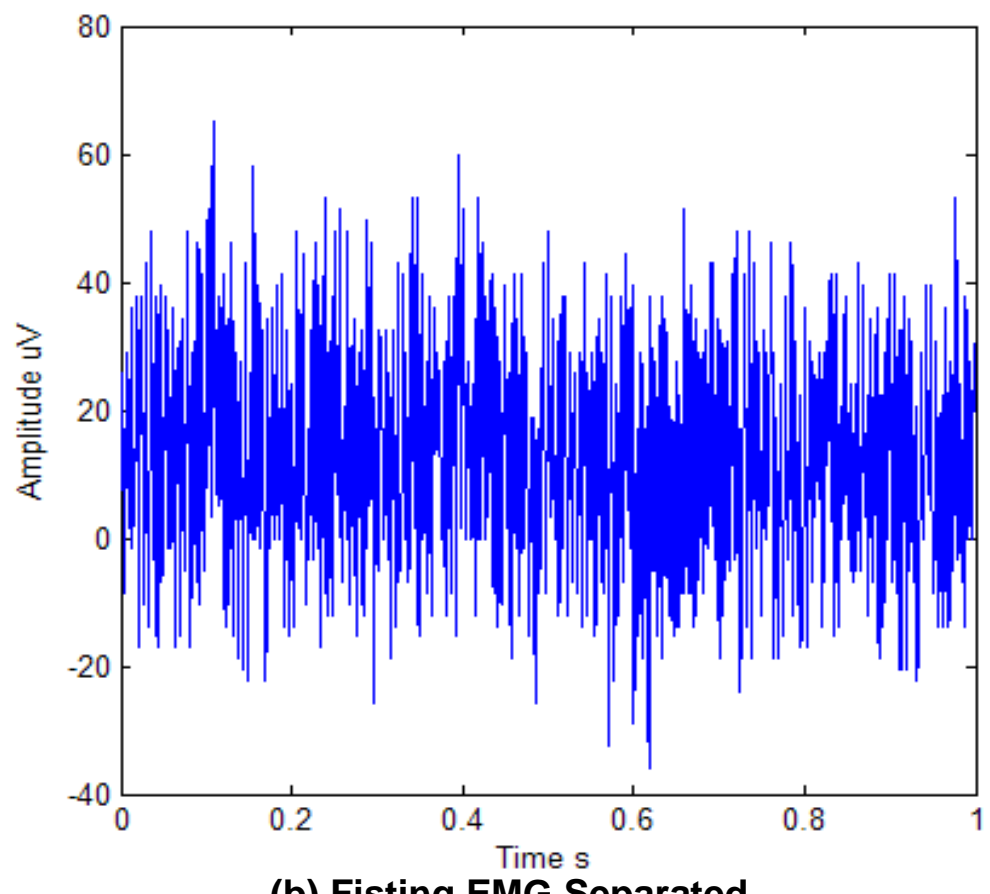

(b) Fisting EMG Separated

Figure 5. EMG Separated

Figure 6 is public poof of EMG before and after the separation, the green power spectrum is the one before filtering, the red power spectrum is the one after filtering. We can see in the Figure 6 that the power spectrum after the separation became smooth about $50 \mathrm{~Hz}, 150 \mathrm{~Hz}$, this proves that this method is very effective to reduce the noises of the power frequency interference in EMG. 


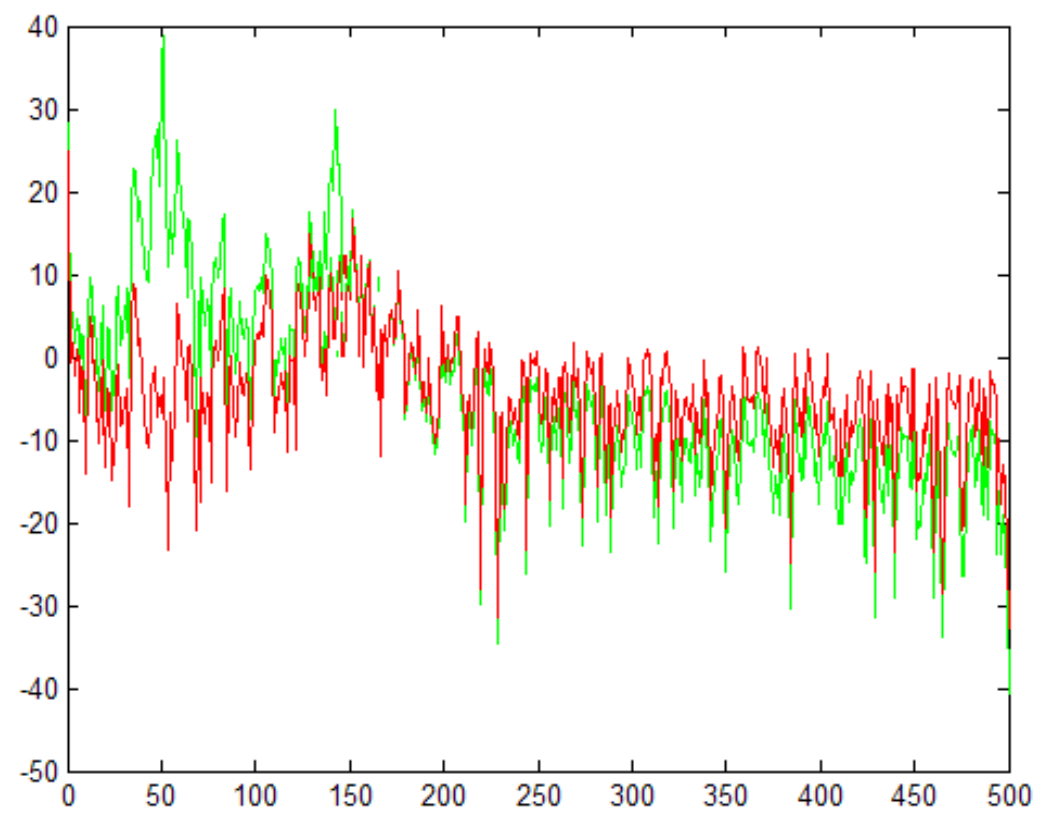

Figure 6. Power Spectrum of EMG of Fisting before and after the Separation

\section{Conclusions}

During the acquisition, SEMG will be influenced by all kinds of noises more or less. And the power frequency and its harmonic interference of $50 \mathrm{~Hz}$ is one of the most important interference source, its amplitude has a 2-3 higher magnitude order than that of EMG, so the EMG collected from the power frequency interference is a type of weak signal collection. We can see from this paper that this method can find out the status of power frequency and its harmonics quickly, and filter the targeted power frequency interference, and cannot decay the useful part of the signal, then avoid the additional distortion of EMG during the noise interference removal. Figure 6 analyses and compares the power spectrum before and after the filtering, and found that this method is very efficient to the interference suppression of the periodic signal. So, this method can also be suitable to the other interference suppression of periodic signal.

\section{References}

[1] M. Pin-gao, L. Zhi-zeng and J. Yu-tao, “A Method of decreasing the power frequency noise from SEMG”, Journal of Hangzhou Dianzi University, J. Hangzhou. China, vol. 28, (2008), pp. 70-73.

[2] W. Wei, L. Chengwang and D. Tao, "Research on frequency domain filtering algorithm for power line interfence in high speed data acquisition”, Journal of Geodesy and Geodynamics, J. China, vol. 31, (2011), pp. 100-104.

[3] Z. Guohua, Z. Zongtan and L. Ming, "Analysis \& depression of disturbance of the mains power supply in design of BCI System”, Computer Measurement \& Control, J. China, vol. 14, (2006), pp. 648-650.

[4] C. Yaguang, Y. Zhongle and C. Xinhao, "The Removal coherent noise from evoked potential”, Journal of South-central College for Nationalities (Nat.Sci), J. Yunnan. China, vol. 16, (1996), pp. 14-17.

[5] D. Xiangfeng, S. Yining and S. Qizhu, "The Methods to eliminate the power-line interference in SEMG detection”, Beijing Biomedical Engineering. J. Beijing. China, vol. 26, (2006), pp. 63-67.

[6] W. Bing, W. Kui and L. Xiaolin, "Comprehensive study on removal of power line interference in EEG", Chinese Journal of Sensors and Actuators J. Beijing. China, vol. 23, (2010), pp. 87-92. 
[7] Z. Liang, J. H. Yang, X. J. Qian, X. Chen, Z. Ye, B. Y. Rong, C. X. Tan and H. Q. Feng, "A Study on instantaneous median frequency of surface EMG signals based on time-frequency distributions”, Space Medicine \& Medical Engineering, vol. 17, no. 1, (2004), pp. 54-58.

[8] X. Xingyu, W. Tao and L. Lin, "Design of portable bioelectricity sensor”, Instrument Technique and Sensor, J. China, vol. 3, (2012), pp. 6-8.

[9] Z. O. Khokhar, Z. G. Xiao and C. Menon, "Surface EMG pattern recognition for real-time control of a wrist exoskeleton”, BioMedical Engineering OnLine, vol. 9, (2010), pp. 1-17.

[10] M. F. Chimeno and R. Pallas-arenyr, "A comprehensive model for power line interference in biopotential maesurement”, IEEE Transactions on Instrumentation and Measurement, J. vol. 49, (2000), pp. 535-540.

[11] Z. Junli, Y. Qinghang and Y. Weili, "Singanl and systerm”, Higher Education Press, (2000). 